\title{
Reconstructing the transmission dynamics of varicella in Japan: An elevation of age at infection
}

\author{
Ayako Suzuki ${ }^{1}$, Hiroshi Nishiura ${ }^{\text {Corresp. } 1}$ \\ ${ }^{1}$ School of Public Health, Kyoto University, Kyoto, Kyoto, Japan \\ Corresponding Author: Hiroshi Nishiura \\ Email address: nishiurah@gmail.com
}

Background. In Japan, routine two-dose immunization against varicella has been conducted among children at ages of 12 and 36 months since 2014, and the vaccination coverage has reached around $90 \%$. To understand the impact of routine varicella vaccination, we reconstructed the epidemiological dynamics of varicella in Japan.

Methods. Epidemiological and demographic datasets over the past three decades were analyzed to reconstruct the number of susceptible individuals by age and year. To estimate the annual risk of varicella infection, we fitted a balance equation model to the annual number of cases from 1990 to 2019. Using parameter estimates, we reconstructed varicella dynamics starting from 1990 and modeled future dynamics until 2033.

Results. Overall varicella incidence declined over time and the annual risk of infection among children younger than 10 years old decreased monotonically starting in 2014. Conversely, varicella incidence among teenagers (age 10 to 14 years) has increased each year since 2014. A substantial number of unvaccinated individuals born before the routine immunization era remained susceptible and aged without contracting varicella, while the annual risk of infection among teenagers aged 10 to 14 years increased starting in 2011 despite gradual expansion of varicella vaccine coverage. The number of susceptible individuals decreased over time in all age groups. Modeling indicated that susceptibility rates among pre-school children aged 1 to 4 years will remain low.

Conclusion. Routine varicella vaccination has successfully reduced infections in pre-school and early primary school age children, but has also resulted in increased infection rates among adolescents. This temporary increase was caused both by the increased age of susceptible individuals and increased transmission risk among adolescents resulting from the dynamic nature of varicella transmission. Monitoring susceptibility among adolescents will be important to prevent outbreaks over the next decade. 


\section{TITLE}

2 Reconstructing the transmission dynamics of varicella in Japan: An elevation of age at infection

3

\section{Authors:}

$5 \quad$ Ayako Suzuki $^{1}$ and Hiroshi Nishiura ${ }^{1 *}$

6

7 Affiliations:

$8{ }^{1}$ Kyoto University School of Public Health, Yoshidakonoe cho, Sakyo-ku, Kyoto City 6068501, 9 Japan

10

$11 *$ Correspondence to:

12 Hiroshi Nishiura

13 Kyoto University School of Public Health

14 Yoshidakonoecho, Sakyo-ku, Kyoto 6068501, Japan

15 Tel: +8175753 4456, Fax: +81757534458

16 E-mail: nishiurah@gmail.com 


\section{Abstract (500 words)}

18 Background. In Japan, routine two-dose immunization against varicella has been conducted among children at ages of 12 and 36 months since 2014, and the vaccination coverage has reached around $90 \%$. To understand the impact of routine varicella vaccination, we reconstructed

21 the epidemiological dynamics of varicella in Japan.

22 Methods. Epidemiological and demographic datasets over the past three decades were analyzed 23 to reconstruct the number of susceptible individuals by age and year. To estimate the annual risk 24 of varicella infection, we fitted a balance equation model to the annual number of cases from 251990 to 2019. Using parameter estimates, we reconstructed varicella dynamics starting from 261990 and modeled future dynamics until 2033.

27 Results. Overall varicella incidence declined over time and the annual risk of infection among 28 children younger than 10 years old decreased monotonically starting in 2014. Conversely, 29 varicella incidence among teenagers (age 10 to 14 years) has increased each year since 2014. A 30 substantial number of unvaccinated individuals born before the routine immunization era

31 remained susceptible and aged without contracting varicella, while the annual risk of infection 32 among teenagers aged 10 to 14 years increased starting in 2011 despite gradual expansion of varicella vaccine coverage. The number of susceptible individuals decreased over time in all age groups. Modeling indicated that susceptibility rates among pre-school children aged 1 to 4 years 35 will remain low.

36 Conclusion. Routine varicella vaccination has successfully reduced infections in pre-school and

37 early primary school age children, but has also resulted in increased infection rates among

38 adolescents. This temporary increase was caused both by the increased age of susceptible

39 individuals and increased transmission risk among adolescents resulting from the dynamic nature 
40 of varicella transmission. Monitoring susceptibility among adolescents will be important to 41 prevent outbreaks over the next decade.

42

\section{Keywords}

44 Chickenpox; Childhood immunization; Epidemiology; Mathematical model; Herd immunity;

45 Transmission dynamics

46 


\section{Introduction}

48 Varicella-zoster virus (VZV) is a human alphaherpesvirus with a double-stranded DNA genome.

49 VZV is the causative agent of varicella (chickenpox) and herpes zoster. Primary infection with

50 VZV results in varicella, which is characterized by an itchy vesicular rash. Varicella causes mild

51 to moderate illness in healthy children but symptoms can be serious, especially in infants,

52 adolescents, adults, and immunocompromised individuals. VZV enters a latent state in dorsal

53 root ganglia and subsequent reactivation causes herpes zoster. Older and immunosuppressed

54 individuals are at increased risk of herpes zoster (Arvin, 1996; Gershon et al., 2015).

55 A live attenuated varicella vaccine, initially derived from the Oka strain, was developed

56 in Japan in 1994 (Takahashi et al., 1974). Prior to the introduction of varicella vaccines, varicella

57 was a universal childhood disease worldwide. A varicella vaccination program was first

58 introduced in the United States in 1995 and varicella incidence declined by $90 \%$ over the next

59 decade (Guris et al., 2008). However, single dose vaccination was considered insufficient to

60 prevent VZV transmission in children, and high numbers of breakthrough infections led to

61 adoption of routine two-dose vaccination starting in 2007 (Lopez et al., 2006; Gao et al., 2009).

62 Based on data from the World Health Organization Vaccine Preventable Diseases Monitoring

63 System, a total of 51 countries and regions had implemented one- or two-dose universal varicella

64 vaccination as of 2021 (World Health Organization, 2018). A meta-analysis of published studies

65 from 1995 to 2014 showed that one-dose and two-dose varicella vaccination was $81 \%$ and $92 \%$

66 effective in preventing varicella, respectively (Marin et al., 2016).

67 Although the varicella vaccine was commercially available in Japan starting in 1987, it

68 was not rapidly integrated into routine vaccination programs and the vaccination rate remained

69 around 40\% during the 1990s (Yoshikawa, Kawamura \& Ohashi, 2016). Subsequently, vaccine 
70 coverage gradually increased starting around 2010 as some local governments initiated subsidy

71 program for varicella vaccination. The Japan Pediatric Society recommended two-dose varicella

72 immunization in 2012. In 2014, varicella vaccination coverage reached around $90 \%$ after a two-

73 dose varicella immunization program for children aged 12 to 36 months was introduced, and by

742019 vaccination had resulted in an $80 \%$ reduction in varicella incidence (National Institute of

75 Infectious Diseases, 2018).

76 Theoretical concerns regarding the introduction of universal varicella vaccination

77 included increased age at infection following mass vaccination and potential for increased

78 incidence of herpes zoster (Gidding et al., 2005; Karhunen et al., 2010). Varicella vaccine is not

79 included as a routine childhood immunization in the United Kingdom as of 2021 (National

80 Health Service, 2019). Varicella in adults can be serious and even result in death in some

81 individuals. The overall case-fatality risk (CFR) of varicella from 1990 to 1994 was 2.6 per

82100,000 infections with the highest CFR observed in those older than 20 years. The CFR among

83 adults aged more than 20 years was $21.3,25$ times higher than that among children aged 12

84 months to 4 years (Meyer et al., 2000). Various modeling studies have been carried out to

85 evaluate the population impact of universal varicella vaccination. Halloran et al. showed that the

86 number of older susceptible children increased after initiation of a varicella vaccination program,

87 and that this effect became more apparent as vaccine coverage increased (Halloran et al., 1994).

88 Many followers assessed the impact of universal varicella immunization on the incidence of

89 varicella (Brisson et al., 2000; Karsai et al., 2000; Pawaskar et al., 2021; Suh et al., 2021; van

90 Hoek et al., 2011).

91 Despite numerous modelling efforts, it remains unclear how the numbers of susceptible

92 individuals of a given age will impact the long-term epidemiological dynamics of varicella. 
93 Almost seven years have elapsed since the initiation of routine varicella vaccination in Japan.

94 Monitoring changes in the fraction of susceptible individuals by birth cohort could help to

95 project future varicella dynamics. The aim of this study was to assess temporal changes in the

96 Japanese population susceptible to varicella. Using reported number of cases from 1990 to 2019,

97 we estimated the risk of infection as a function of time and age and reconstructed

98 epidemiological dynamics until 2033.

99 Materials \& Methods

100 Epidemiological data

101 Three different types of data were used: (i) age-specific annual incidence of varicella (i.e., annual

102 number of notified chickenpox cases by age group), (ii) vaccination coverage, and (iii) number

103 of newborns. Varicella incidence was based on notifications to the National Epidemiology

104 Surveillance for Infectious Diseases (NESID) system in compliance with the Communicable

105 Disease Prevention Law until March 1999 and subsequently the Infectious Diseases Control

106 Law. Varicella case was defined as presentation to medical services with the following

107 symptoms: (1) sudden generalized development of serous papules and vesicles and (2) co-

108 existence of rashes in different stages (papules, vesicles, and crusts) (Ministry of Health, Labour

109 and Welfare of Japan, 2021a). Varicella is classified as a category V disease, a subset of which

110 including varicella are monitored by sentinel surveillance systems. Varicella is a pediatric

111 disease in Japan, and physicians at approximately 3,000 pediatric sentinel sites (representing

112 approximately $10 \%$ of pediatric clinics and hospitals in Japan) notified cases to NESID on a

113 weekly basis. Reporting coverage in the NESID system (i.e., the proportion of notified cases

114 included in surveillance out of all infections) was calculated as the cumulative number of cases 
115 divided by the number of newborns in each cohort. Since the surveillance system was revised

116 along with the change in law in April 1999, reporting coverage was separately calculated before

117 and after 1999 (see Supplementary Figure S1).

118 Routine assessment of varicella vaccination coverage was not conducted before the

119 introduction of the routine varicella vaccination program in 2014. Instead, we used vaccination

120 coverage estimated from annual vaccine sales divided by numbers of births in the previous year

121 (Ozaki., 2013). Vaccination coverage after 2014 was extracted from the database of the National

122 Epidemiological Surveillance of Vaccine-Preventable Diseases (NESVPD), which regularly

123 investigates the seroprevalence and vaccination rates for infectious diseases subject to routine

124 vaccination (Ministry of Health, Labour and Welfare of Japan, 2021b).

125 To model the future dynamics of varicella in Japan, we used the number of newborns

126 after 2021 from the Population Projections for Japan (2017) from 2016 to 2065 (National

127 Institute of Population and Social Security Research, 2017).

\section{Mathematical model}

129 In the following model, we consider the depletion of susceptible individuals over the course of

130 life using a mathematical model. Assuming that all unvaccinated individuals eventually contract

131 varicella, our model considers that recruitment of new susceptible individuals occurs through

132 births (Finkenstädt \& Grenfell, 2000; Wallinga, Teunis \& Kretzschmar, 2002) and that the size

133 of the susceptible proportion of a given birth cohort subsequently declines either by vaccination

134 or infection. The model imposes a few simplistic assumptions: (i) the first dose of varicella

135 vaccine confers full protection by adulthood, (ii) following natural infection, acquired immunity

136 persists until adulthood, and (iii) susceptible individuals experience time- and age-dependent

137 annual risks of infection. 


$$
\begin{gathered}
S_{t+1,1}=\left(1-v_{t}\right) B_{t}-I_{t, 1} \text { for the first two years of life }(i=1) \\
S_{t+1, i}=S_{t, i-1}-I_{t, i-1} \quad \text { for } i>1,
\end{gathered}
$$

139 where $t=0,1,2, \ldots$ and $i=1,2,3, \ldots$ denote the calendar year starting from 1990 and

140 chronological age (in years), respectively. $S_{t, i}$ is the number of susceptible individuals in year $t$

141 and of age $i, v_{t}$ is the vaccination coverage in year $t, B_{t}$ is the number of newborns in year $t$, and

$142 I_{t, i}$ represents the number of cases in year $t$ of age $i$. Using the number of susceptible individuals,

143 the yearly reported number of new infections, $C_{t+1, i}$, is described by:

$$
C_{t+1, i}=\delta_{t} I_{t+1, i}=\delta_{t} \gamma_{t, i} S_{t, i}
$$

144 where $\delta_{t}$ is the ascertainment factor in year $t$ and $\gamma_{t, i}$ is the annual risk of infection among

145 susceptible individuals in year $t$ and of age $i$. Here, the ascertainment factor has been commonly

146 referred to as "reporting rate" by time-series susceptible-infectious-recovered (TSIR) models

147 (Finkenstadt \& Grenfell, 2000). This is not precisely the reporting rate in that case numbers in

148 surveillance report is not divided by actual number of cases (Ciofi degli Atti et al., 2002;

149 Marziano et al., 2018), but the actual reporting rate in Japan is well known as difficult to be 150 estimated explicitly, due to non-random distribution of medical and healthcare facilities that 151 agreed to cooperate surveillance. In the above model, vaccination coverage $v_{t}$ and the number of newborns $B_{t}$ are directly

153 informed by empirical data, while $S_{t, i}$ and $I_{t, i}$ are reconstructed by estimating parameters $\delta_{t}$ and

$154 \gamma_{t, i}$. First, the reporting coverage $\delta_{t}$ was estimated using a linear regression analysis of the yearly 155 number of newborns and cumulative number of reported cases in the corresponding birth cohort 156 (see Supporting Material). Because the reporting system drastically changed in 1999 when the 
157 notification rules and corresponding laws were revised, reporting coverage was estimated using

158 a step function with parameters $\delta_{A}$ and $\delta_{B}$ representing the periods 1984-1999 and 2000-2015,

159 respectively. Second, maximum likelihood estimation was conducted to estimate the annual risk

160 of infection $\gamma_{t, i}$. We assumed that the annual risk of infection changed every 5 years until 2009,

161 then changed every 2 years after 2010 as the vaccination program was gradually expanded. To

162 construct the likelihood function to estimate $\gamma_{t, i}$, age-specific annual incidence was assumed to

163 follow a Poisson distribution.

$$
L\left(\theta ; \boldsymbol{r}_{\boldsymbol{t}, \boldsymbol{i}}\right)=\prod_{i} \prod_{t} \frac{E\left(C_{t, i}\right)^{r_{t, i}} \exp \left(-E\left(C_{t, i}\right)\right)}{r_{t, i} !}
$$

164 where $\mathrm{E}($.$) represents the expected number and r_{\mathrm{t}, \mathrm{i}}$ is the reported number of cases in year $t$ and 165 age group $i$.

166 Using parameter estimates, we reconstructed the number of susceptible individuals over

167 time and by age. Subsequently, we computed the projected epidemiological dynamics of

168 varicella until 2033. To account for parameter uncertainties, the $95 \%$ confidence intervals of

169 projected notification rates were computed using the parametric bootstrap method.

170 Data sharing statement

171 Annual varicella surveillance data by age group and demographic cohort of newborns are

172 available as Online Supporting Material (Supplementary Tables 1 and 2).

\section{Ethical considerations}

174 The present study used publicly available information. Because no private information was used,

175 ethical approval was not required. 


\section{Results}

177 Figure 1A shows the age distribution of reported varicella cases from 1990 to 2019. The overall

178 number of reported varicella cases declined by $76 \%$ from 2011 to 2019 . Although incidence

179 among preschool age children (age 1 to 4 years) continued to decrease after 2011, a similar

180 decline was not observed among school-aged children. Varicella incidence among children aged

1815 to 9 years decreased from 2011 to 2015, but it did not show a declining trend after 2015

182 (Figure 1B). Interestingly, varicella incidence among teenagers aged 10 to 14 years increased

183 every year after the initiation of a routine immunization program in 2014 (Figure 1C). Varicella

184 vaccine coverage gradually but steadily increased over time, with an abrupt increase observed

185 after 2014. Although varicella vaccine coverage was only around $20 \%$ in the 1990 s, coverage

186 reached $90 \%$ by 2017 (Figure 2).

187 Figures 3 shows the estimated annual risk of infection among susceptible individuals by

188 age group and year. The annual risk of infection among pre-school children aged 1 to 4 years

189 decreased starting in 2011. Declining annual risks of infection over time were subsequently

190 observed among school-age children (age 5 to 9 years) after 2015. Conversely, the annual risk of

191 infection among teenagers aged 10 to 14 years increased after 2011 (Supplementary Figure S2).

192 The risk of infection was highest among 4-year-old children until 2013. After this time, the age

193 of peak varicella risk increased, and in 2018/2019 the age group with the highest risk of infection

194 became teenagers aged 10 to 14 years.

195 Comparisons of the observed and estimated numbers of reported varicella cases are

196 shown in Figure 4. While our model was kept very simple and tractable, it captured the

197 empirically observed overall patterns of infection in all age groups, including those with both

198 increasing and decreasing numbers of cases over time. 
Figures 5A and 5B illustrate depletion of the susceptible population with advancing age,

200 stratified by birth year. The susceptible population decreased over time, and the age-dependent

201 decline in susceptibility was accelerated in pre-school age children following the introduction of

202 routine immunization program. Figures 5C, 5D and 5E show the long-term dynamics of the

203 susceptible population by age over the past three decades and until 2033. Although the number

204 of susceptible pre-school age children (age 1 to 4 years) decreased over time and remained at low

205 level, numbers of susceptible children aged 5 years and older temporarily increased shortly after

206 the initiation of routine immunization in 2014 (Figure 5D). In older age groups, increased

207 numbers of susceptible individuals were observed at later times (Figures 5D and 5E). For

208 example, the number of unvaccinated susceptible individuals showed a sharp peak among

209 children aged 5 years in 2018. Aging of this unvaccinated birth cohort is seen every year, as

210 illustrated by the damped, yet sharp, peak in 2023 for 10-year-old children.

\section{Discussion}

212 The present study evaluated the impact of the routine varicella immunization program in Japan

213 on long-term varicella epidemiological dynamics using demographic and surveillance data as

214 well as information on vaccination coverage. We first estimated the age- and time-dependent

215 annual risks of infection among susceptible individuals. Using parameter estimates, we

216 reconstructed and predicted varicella dynamics in Japan from 1990 to 2033. Our model

217 successfully captured the age shift in susceptibility to varicella infection that occurred along with

218 increased vaccine coverage; however, the most remarkable age shift in the susceptible population

219 occurred via aging of the unvaccinated birth cohort. Following introduction of the routine

220 immunization program, the risk of infection gradually increased among teenagers as

221 unvaccinated susceptible individuals accrued in the corresponding age groups. The elevated age 
222 at infection has been observed in other countries including the USA (Halloran et al., 1994), and

223 what is unique to Japan is that such change has been seen in a drastic manner following an

224 introduction of universal vaccination program.

225 Two critical points should be taken from our study. Firstly, widespread vaccination had

226 an immense impact on the epidemiological dynamics of varicella. The overall incidence of

227 varicella sharply declined, even before the initiation of routine vaccination when vaccination was

228 voluntary and sporadically covered local government subsidies. The unvaccinated birth cohort

229 who had never contracted varicella remained susceptible and aged without contracting varicella.

230 Using modeling techniques, we successfully reconstructed the dynamics of the susceptible

231 population over time. The number of susceptible individuals decreased in all age groups starting

232 in the 1990s. However, there was a short-term increase in the number of susceptible children

233 under 5 years of age shortly after the introduction of routine vaccination. Subsequently, a short-

234 term increase in the number of susceptible individuals was observed in older age groups.

235 Although susceptibility eventually decreased, many unvaccinated individuals born from 2010 to

2362014 (i.e., shortly before implementation of the routine immunization program) remained

237 susceptible until their teenage years. Our model projected that this over-represented susceptible 238 cohort may remain identifiable until around 2027.

239 Secondly, it should be noted that the annual risk of infection increased with age,

240 especially among school-age children. The annual risk of infection among children aged 5 to 9

241 years elevated during the period of varicella vaccine expansion around 2010, which was not in

242 line with declining birth rate and subsidized vaccination (at moderate coverage) at local

243 municipality levels. We speculate that the number of susceptible population increased before

2442014 among children aged 5-9 and this increase in susceptibility resulted in the increased risk of 
245 infection among them. A published study from France that explored pre-school children

246 attributed similar increase to an increased social mixing among pre-school children (Marziano et

247 al., 2018). The annual risk of infection among children aged 5 to 9 years subsequently declined

248 following implementation of the routine vaccination program starting in 2014. Among teenagers,

249 annual risk of infection has continued to increase since 2010. The annual risk of infection can

250 increase either by increased transmissibility (or transmission rate) or increased prevalence of

251 infection. Considering that the age of peak susceptibility temporarily increased during the period

252 of varicella vaccine expansion, we speculate that an increase in the number of infectious

253 individuals resulted in the elevated risk of infection among teenagers (e.g., increased chance of

254 transmission in school settings).

255 The accumulation of susceptible adolescents implies the potential for varicella outbreaks

256 in middle or high schools in the near future in Japan. Modeling has also suggested the potential

257 for post-honeymoon epidemics for other vaccine preventable diseases such as measles (Metcalf

258 et al., 2020). In the case of varicella, school outbreaks among highly vaccinated populations have

259 been reported in the United States, where two-dose routine vaccination was implemented in 2007

260 (Gao et al., 2009; Lopez et al., 2006). Although most outbreaks were observed among children

261 aged 5 to 9 years, several outbreaks occurred among high school students who are at risk for

262 severe varicella infection (Boëlle \& Hanslik, 2002). A study analyzing active surveillance data

263 for varicella outbreaks from 2012 to 2015 concluded that most outbreak-associated infections

264 occurred in under-vaccinated children who were unvaccinated or received only single-dose

265 vaccination (Lopez et al., 2019). The same study found that outbreak sizes were smaller among

266 students because of the high proportion of two-dose vaccine recipients. 

systematic review of studies published from 1974 to 2016 concluded that severe breakthrough varicella is rare, but can occur (Leung et al., 2017). To avoid varicella outbreaks as well as severe infection, ensuring primary and/or booster vaccination of adolescents and young adults

271 may need to be considered at some point in the future, as was the case for rubella to minimize 272 risk of congenital rubella syndrome (Kinoshita \& Nishiura., 2016). The observed phenomena 273 was likely associated with the increased clinical severity of varicella among teenagers compared 274 with younger children, while vaccination coverage among adolescent remained insufficient.

275 Regular observation of seroepidemiological data is also vital to identify birth cohorts at high risk 276 of varicella infection. If the cost associated with adolescent cases is substantial, or if the country 277 aims to eliminate varicella, the possible solution would include supplementary immunization 278 program among adolescents. To accomplish objective judgement of such policy option, regular 279 monitoring of susceptibility, especially among unvaccinated birth cohorts not subjected to two280 dose universal vaccination, will be required for the next several decades.

282 model, and the model structure may have been oversimplified. There are inevitable trade-offs 283 between model complexity and potential to provide biological insights. Despite its simplistic 284 nature, the model used in the present study successfully captured the aging of unvaccinated susceptible individuals and increased risks of infection among adolescents, allowing future

286 projections of varicella dynamics. Secondly, we assumed that single-time varicella vaccination in 287 the model successfully prevented infection. One randomized controlled study comparing single288 dose and two-dose varicella vaccination reported that vaccine efficacy after 10-year follow-up 289 was $94.4 \%$ for single-dose and $98.3 \%$ for two-dose vaccination (Kuter et al., 2004). Thirdly, we 
290 did not consider waning immunity over time since vaccination. Duration of protection to

291 varicella is not well understood (SAGE Working Group on Varicella and Herpes Zoster

292 Vaccines, 2014). However, a study conducted during the period of single-dose vaccination in the

293 United States showed that the risk of moderate or severe varicella among children who had

294 received varicella vaccines at least 5 years previously was 2.6 times higher than among children

295 who had been vaccinated less than 5 years previously (Chaves et al., 2007).

296 The present study did not investigate the epidemiology of herpes zoster. An increased

297 incidence of herpes zoster following initiation of the universal varicella vaccination program has

298 been reported in Japan (Toyama \& Shiraki, 2018). Toyama et al. concluded that declining

299 varicella incidence among children reduced exogenous boosting events among their parents. As a

300 result, herpes zoster incidence increased among adults, consistent with the Hope-Simpson

301 hypothesis (Hope-Simpson, 1965; Thomas, Wheeler \& Hall, 2002). Another possible

302 explanation for the increase in herpes zoster incidence in Japan is the consequence of the

303 declining in birth rate and the ageing society. Modeling study conducted in Spain (Marziano et

304 al., 2015) and Germany (Horn et al., 2018) indicated that the demographic changes reduced the

305 opportunities exogenous boosting, resulted in the increase in herpes zoster incidence, especially

306 in elderly population. Future studies on this subject could explore these possible mechanisms

307 along with empirical data.

The present study did not account for the potential effects of public health and hygiene

309 measures implemented in response to the coronavirus disease 2019 pandemic on varicella

310 dynamics. A study using a time-series Susceptible-Infected-Recovered (TSIR) model indicated

311 the potential for future large outbreaks of endemic diseases, such as respiratory syncytial virus

312 (RSV) and seasonal influenza, because of increasing numbers of susceptible individuals during 
313 controlled periods (Baker et al., 2020). In line with this hypothesis, increased numbers of RSV

314 infections were reported in the 2021 season in Tokyo, Japan (Ujiie et al., 2021). Similar effects

315 may be observed for other infectious diseases, including varicella. This possibility will need to

316 be examined in a future study.

317 While significant future work remains, the present study successfully evaluated the

318 impact of routine varicella immunization on transmission dynamics in Japan. Direct and indirect

319 effects of vaccination drastically reduced varicella incidence, but the program resulted in

320 subgroups of unvaccinated susceptible individuals and increased annual risks of infection among

321 adolescents, indicating the potential for future outbreaks. Regular monitoring of susceptibility,

322 especially among unvaccinated birth cohorts not subjected to two-dose universal vaccination,

323 will be required for the next several decades.

324

\section{Funding}

326 This study was supported by funding from Health and Labor Sciences Research Grants

327 (19HB1001, 19HA1003, 20CA2024, 20HA2007, and 21HB1002 to H.N.), the Japan Agency for

328 Medical Research and Development JP20fk0108140 and JP21fk0108612 to H.N.), the Japan

329 Society for the Promotion of Science KAKENHI (A.S: 19K24150, H.N: 17 H04701 and

330 21H03198), the Inamori Foundation, the GAP Fund Program of Kyoto University, the Japan

331 Science and Technology Agency CREST program (JPMJCR1413 to H.N.), and the SICORP

332 program (JPMJSC20U3 and JPMJSC2105 to H.N.). The funders played no role in the study

333 design, data collection and analysis, decision to publish, or preparation of the manuscript.

334 Acknowledgments 
335 We thank Edanz Group (https://en-author-services.edanz.com/ac) for editing a draft of this

336 manuscript.

337 Conflict of interest

338 We declare that we have no conflict of interest.

339

340 References

341

342

343

344

345

346

347

348

349

350

351

352

353

354

355

356

357

358

359

360

361

362

363

364

365

366

367

368

369

370

371

Arvin AM. 1996. Varicella-zoster virus. Clinical Microbiology Reviews. 9:361-381.

Baker RE, Park SW, Yang W, Vecchi GA, Metcalf CJE, Grenfell BT. 2020. The impact of COVID-19 nonpharmaceutical interventions on the future dynamics of endemic infections. Proceedings of the National Academy of Sciences of the United States of America. 117:30547-30553.

Boëlle PY, Hanslik T. 2002 Varicella in non-immune persons: Incidence, hospitalization and mortality rates. Epidemiology and Infection. 129:599-606.

Brisson M, Edmunds WJ, Gay NJ, Law B, De Serres G. 2000. Modelling the impact of immunization on the epidemiology of varicella zoster virus. Epidemiology and Infection. 125:651-669.

Chaves SS, Gargiullo P, Zhang JX, Civen R, Guris D, Mascola L, Seward JF. 2007 Loss of vaccine-induced immunity to varicella over time. New England Journal of Medicine. 356:11211129.

Ciofi degli Atti ML, Rota MC, Mandolini D, Bella A, Gabutti G, Crovari P, Salmaso S. 2002. Assessment of varicella underreporting in Italy. Epidemiology and Infection 128: 479-484.

Finkenstädt B, Grenfell B. 1998. Empirical determinants of measles metapopulation dynamics in England and Wales. Proceeding of the Royal Society B: Biological Sciences. 265:211-220.

Finkenstädt B, Grenfell B. 2002. Dynamics of measles epidemics: Estimating scaling of transmission rates using a time series SIR model. Ecological Monographs 72: 169-184.

Gao Z, Gidding HF, Wood JG, MacIntyre CR. 2010. Modelling the impact of one-dose vs. twodose vaccination regimens on the epidemiology of varicella zoster virus in Australia. Epidemiology and Infection. 138:457-468.

Peer) reviewing PDF | (2021:10:66298:1:0:NEW 9 Dec 2021) 
372 Gershon AA, Breuer J, Cohen JI, Cohrs RJ, Gershon MD, Gilden D, Grose C, Hambleton S,

373 Kennedy PGE, Oxman MN, Seward JF, Yamanishi K. 2015. Varicella zoster virus

374 infection. Nature Reviews Disease Primers. 1:15016.

375

376

377

378

379

380

381

382

383

384

385

386

387

388

389

390

391

392

393

394

395

396

397

398

399

400

401

402

403

404

405

406

407

408

409

410

411

412

413

414

415

416

Gidding HF, Brisson M, Macintyre CR, Burgess MA. 2005. Modelling the impact of vaccination on the epidemiology of varicella zoster virus in Australia. Australian and New Zealand Journal of Public Health. 29:544-551.

Guris D, Jumaan AO, Mascola L, Watson BM, Zhang JX, Chaves SS, Gargiullo P, Perella D, Civen R, Seward JF. 2008. Changing varicella epidemiology in active surveillance sites-United States, 1995-2005. The Journal of Infectious Diseases. 197 Suppl 2:S71-S75.

Halloran ME, Cochi SL, Lieu TA, Wharton M, Fehrs L. 1994. Theoretical epidemiologic and morbidity effects of routine varicella immunization of preschool children in the United States. American Journal of Epidemiology. 140:81-104.

Hope-Simpson RE. 1965. The nature of herps zoster: A long-term study and a new hypothesis. Proceedings of the Royal Society of Medicine. 58:9-20.

National Institute of Infectious Diseases. 2018. Infectious Diseases Surveillance System in Japan. Available at https://www.niid.go.jp/niid/images/epi/nesid/nesid_en.pdf (accessed 29 September 2021).

Karhunen M, Leino T, Salo H, Davidkin I, Kilpi T, Auranen K. 2010. Modelling the impact of varicella vaccination on varicella and zoster. Epidemiology and Infection. 138:469-481.

Karsai J, Csuma-Kovács R, Dánielisz Á, Molnár Z, Dudás J, Borsos T, Röst G. 2020. Modeling the transmission dynamics of varicella in Hungary. Journal of Mathematics in Industry. 10:12.

Kinoshita R, Nishiura H. Assessing herd immunity against rubella in Japan: A retrospective seroepidemiological analysis of age-dependent transmission dynamics. 2016. BMJ Open. 6:e009928.

Kuter B, Matthews H, Shinefield H, Black S, Dennehy P, Watson B, Resinger K, Kim LL, Lupinacci L, Hartzel J, Chan I, Study Group for Varivax. 2004. Ten year follow-up of healthy children who received one or two injections of varicella vaccine. The Pediatric Infectious Disease Journal. 23:132-137.

Lopez AS, Guris D, Zimmerman L, Gladden L, Moore, T, Haselow DT, Loparev VN, Schmid DS, Jumaan AO, Snow SL. 2006. One dose of varicella vaccine does not prevent school outbreaks: Is it time for a second dose? Pediatrics. 117:e1070-e1077.

Lopez AS, Zhang J, Marin M. 2016. Epidemiology of varicella during the 2-dose varicella vaccination program - United States, 2005-2014. Morbidity and Mortality Weekly Report. 65:902-905.

417 
418 Lopez AS, LaClair B, Buttery V, Zhang Y, Rosen J, Taggert E, Robinson S, Davis M, Waters C, 419 Thomas CA, Rodriguez C, Thomas E, Tuttle J, Brantley T, Perella D, Del Rosario M, Marin M. 420 2019. Varicella outbreak surveillance in schools in sentinel jurisdictions, 2012-2015. Journal of 421 the Pediatric Infectious Disease Society. 8:122-127.

422

423

424

425

426

427

428

429

430

431

432

433

434

435

436

437

438

439

440

441

442

443

444

445

446

447

448

449

450

451

452

453

454

455

456

457

458

459

460

Horn J, Damm O, Greiner W, et al. Influence of demographic changes on the impact of vaccination against varicella and herpes zoster in Germany - a mathematical modelling study. BMC Med. 2018;16(1):3. Published 2018 Jan 9.

Marin M, Marti M, Kambhampati A, Jeram SM, Seward JF. 2016. Global varicella vaccine effectiveness: A meta-analysis. Pediatrics. 137:e20153741.

Marziano V, Poletti P, Guzzetta G, Ajelli M, Manfredi P, Merler S. The impact of demographic changes on the epidemiology of herpes zoster: Spain as a case study. Proc Biol Sci.

2015;282(1804):20142509.

Marziano V, Poletti P, Béraud G, Boëlle PY, Merler S, Colizza V. 2018. Modeling the impact of changes in day-care contact patterns on the dynamics of varicella transmission in France between 1991 and 2015. PLoS Computational Biology 14: e1006334.

Meyer PA, Seward JF, Jumaan AO, Wharton M. 2000. Varicella mortality: Trends before vaccine licensure in the United States, 1970-1994. The Journal of Infectious Diseases. 182:383390.

Metcalf CJE, Wesolowski A, Winter AK, Lessler J, Cauchemez S, Moss WJ, McLean AR, Grenfell BT. 2020. Using serology to anticipate measles post-honeymoon period outbreaks. Trends in Microbiology. 28:597-600.

Ministry of Health, Labour and Welfare of Japan. 2021a. Notification rules of infectious diseases: Chickenpox. Available at https://www.mhlw.go.jp/bunya/kenkou/kekkakukansenshou11/01-05-19.html (accessed 29 September 2021).

Ministry of Health, Labour and Welfare of Japan. 2021b. Procedure for the National Epidemiological Surveillance of Vaccine-Preventable Diseases. Available at https://www.mhlw.go.jp/content/10601000/000645265.pdf(accessed 29 September 2021).

National Health Service. 2019. Chickenpox vaccine overview. Available at https://www.nhs.uk/conditions/vaccinations/chickenpox-vaccine/(accessed 29 September 2021).

National Institute of Population and Social Security Research. 2017. Population Projections for Japan (2017): 2016 to 2065. Available at http://www.ipss.go.jp/ppzenkoku/e/zenkoku_e2017/pp29_summary.pdf(accessed 29 September 2021).

Ozaki T. 2013. Long-term clinical studies of varicella vaccine at a regional hospital in Japan and proposal for a varicella vaccination program. Vaccine. 31:6155-6160.

Peer) reviewing PDF | (2021:10:66298:1:0:NEW 9 Dec 2021) 
464 Pawaskar M, Burgess C, Pillsbury M, Wisløff T, Flem E. 2021. Clinical and economic impact of

465

466

467

468

469

470

471

472

473

474

475

476

477

478

479

480

481

482

483

484

485

486

487

488

489

490

491

492

493

494

495

496

497

498

499

500

501

502

503

504

505 universal varicella vaccination in Norway: A modeling study. PLoS One. 16:e0254080.

SAGE Working Group on Varicella and Herpes Zoster Vaccines. 2014. Systematic review of available evidence on effectiveness and duration of protection of varicella vaccines. Available at https://www.who.int/immunization/sage/meetings/2014/april/4_Systematic_review_on_effectiv eness_and_duration_of_protection_of_varicella_vaccines.pdf(accessed 29 September 2021).

Suh J, Lee T, Choi JK, Lee J, Park SH. 2021. The impact of two-dose varicella vaccination on varicella and herpes zoster incidence in South Korea using a mathematical model with changing population demographics. Vaccine. 39:2575-2583.

Takahashi M, Otsuka T, Okuno Y, Asano Y, Yazaki T. 1974. Live vaccine used to prevent the spread of varicella in children in hospital. Lancet. 2:1288-1290.

Thomas SL, Wheeler JG, Hall AJ. 2002. Contacts with varicella or with children and protection against herpes zoster in adults: a case-control study. Lancet. 360:678-682.

Toyama N, Shiraki K; Miyazaki Dermatologist Society. 2018. Universal varicella vaccination increased the incidence of herpes zoster in the child-rearing generation as its short-term effect. Journal of Dermatological Science. 92:89-96.

Ujiie M, Tsuzuki S, Nakamoto T, Iwamoto N. Resurgence of respiratory syncytial virus infections during COVID-19 pandemic, Tokyo, Japan. 20201. Emerging Infectious Diseases. 27:10.3201/eid2711.211565.

van Hoek AJ, Melegaro A, Zagheni E, Edmunds WJ, Gay N. 2011. Modelling the impact of a combined varicella and zoster vaccination programme on the epidemiology of varicella zoster virus in England. Vaccine. 29:2411-2420.

Wallinga J, Teunis P, Kretzschmar M. 2003. Reconstruction of measles dynamics in a vaccinated population. Vaccine. 21:2643-2650.

World Health Organization. 2018. WHO vaccine-preventable diseases: Monitoring system. Available at http://apps.who.int/immunization_monitoring/globalsummary/schedules (accessed 29 September 2021).

Yoshikawa T, Kawamura Y, Ohashi M. 2016. Universal varicella vaccine immunization in Japan. Vaccine. 34:1965-1970. 
506 Figure legends

507

508 Figure 1: Number of reported varicella cases in Japan, 1990-2019.

509 (A) Number of reported varicella cases by age. (B, C) Number of reported cases in individuals

510 aged 5 to 9 years $(\mathrm{B})$ and 10 to 14 years $(\mathrm{C})$.

511

512 Figure 2: Estimated varicella vaccine coverage in Japan, 1990-2019.

513 The solid line shows estimated varicella vaccine coverage and dotted horizontal lines show the 5-

514 year average used for data analysis. The dotted vertical line indicates the year (2014) when

515 universal varicella vaccine was implemented.

$516 *$ Estimated based on annual vaccine sales (vaccine doses sold/number of newborns in previous

517 year)

$518 * *$ Extracted from National Epidemiological Surveillance of Vaccine-Preventable Diseases

519 (NESVPD) database

520

521 Figure 3: Estimated annual risk of varicella infection among susceptible individuals.

522 Estimated annual risk of infection stratified by year.

523

524

525

Figure 4: Comparison of observed and estimated numbers of reported varicella cases by

526 age. 
527 Black dots show numbers of reported cases per 100,000 population. Blue lines show estimated

528 numbers of reported cases per 100,000 population and blue error bars indicate $95 \%$ confidence

529 intervals.

530

531 Figure 5: Temporal dynamics of the varicella-susceptible population.

532 (A) Estimated number of susceptible individuals stratified by birth year. (B) Estimated

533 proportion of susceptible individuals (estimated number of susceptible individuals/number of

534 newborns in birth cohort) stratified by birth year. (C-E) Estimated number of susceptible

535 individuals by age. Vertical dotted lines indicate the last year for the analyzed datasets (2019).

536

537 Supplementary Figure S1: Cumulative varicella cases versus cumulative births.

538 To estimate the ascertainment factor, we conducted a regression analysis of the cumulative

539 number of

540 yearly cases versus the cumulative number of yearly births. We calculated the ascertainment

541 factor separately before and after 1999 because of changes in the reporting system in this year.

542 To consider the impact of varicella vaccination, we excluded the vaccinated population from

543 cumulative births ((1-vaccination coverage $) \times$ number of births $)$.

544 (A) Regression analysis using datasets from 1985 to 1999. (B) Regression analysis using

545 datasets from 2000 to 2014.

546

547 Supplementary Figure S2: Estimated annual risk of varicella infection among susceptible

548 individuals. 
549 Estimated annual risk of infection stratified by age. 


\section{Figure 1}

Figure 1: Number of reported varicella cases in Japan, 1990-2019.

(A) Number of reported varicella cases by age. (B, C) Number of reported cases in individuals aged 5 to 9 years (B) and 10 to 14 years (C). 


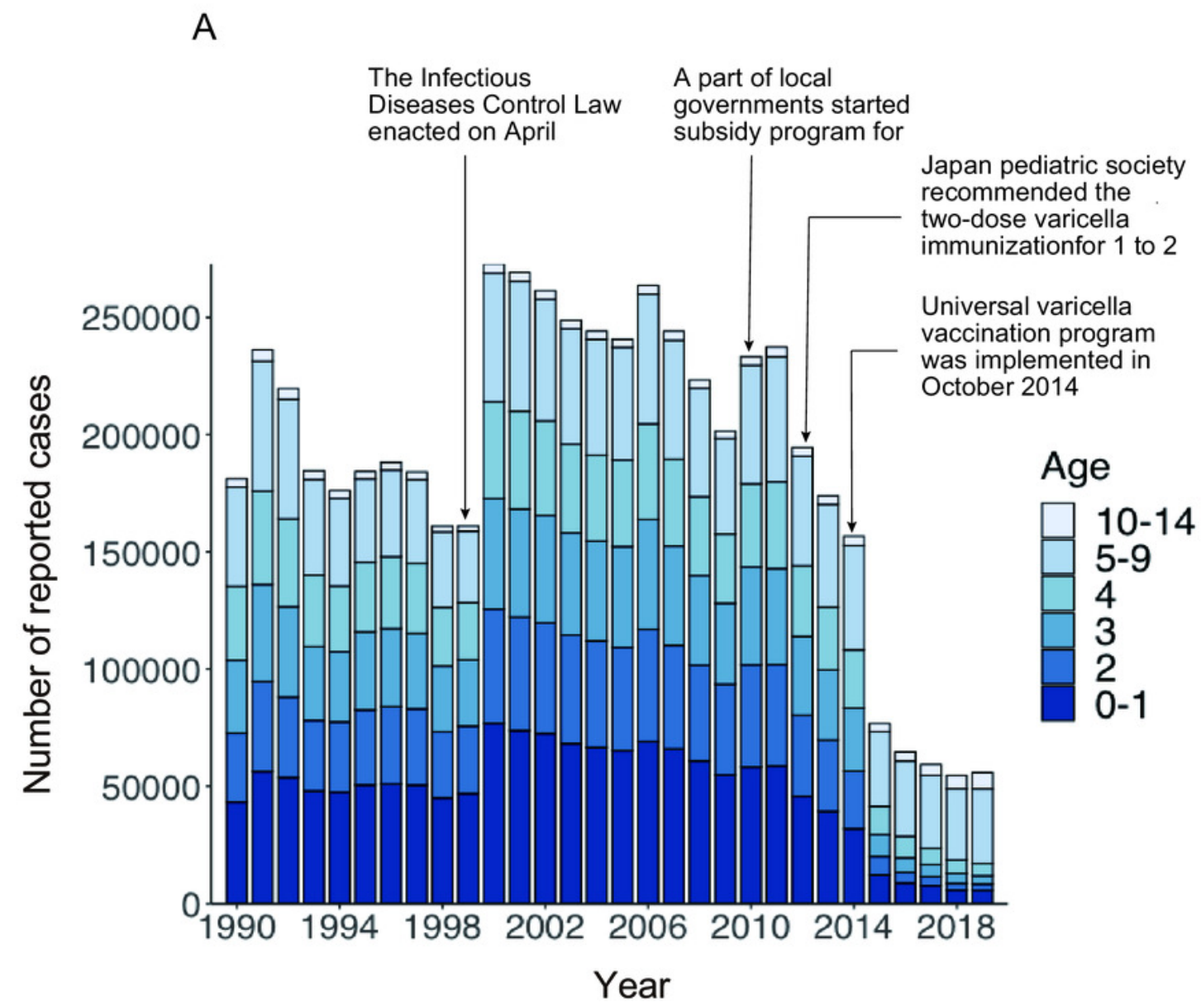

B

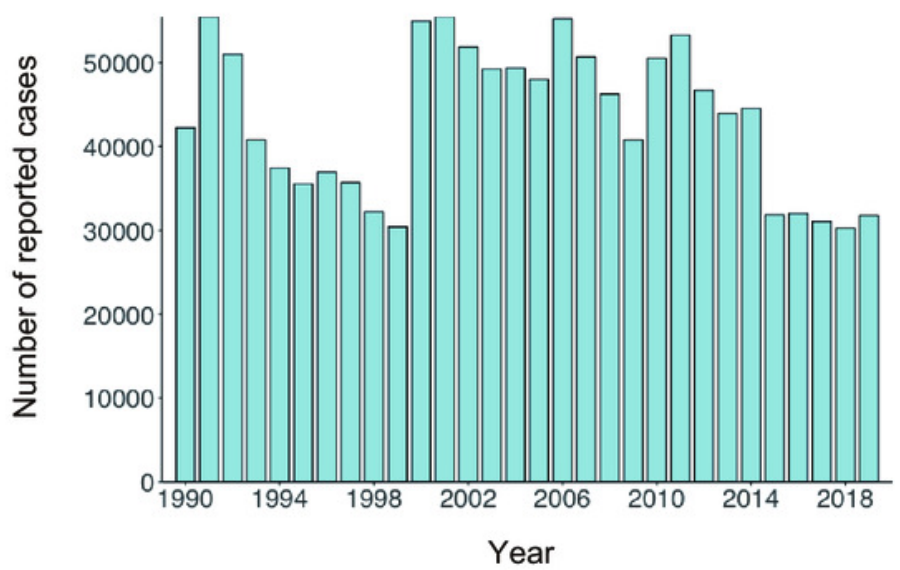

C

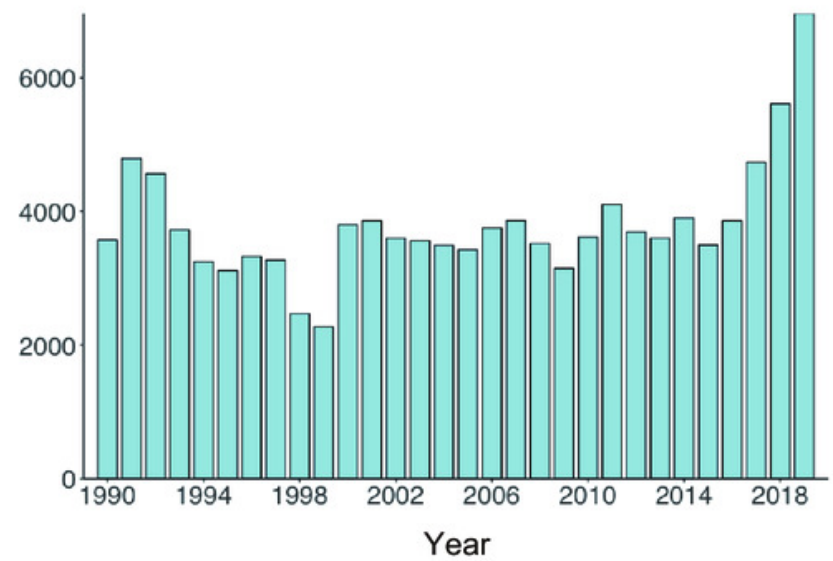


Figure 2

Figure 2: Estimated varicella vaccine coverage in Japan, 1990-2019.

The solid line shows estimated varicella vaccine coverage and dotted horizontal lines show the 5-year average used for data analysis. The dotted vertical line indicates the year (2014) when universal varicella vaccine was implemented. * Estimated based on annual vaccine sales (vaccine doses sold/number of newborns in previous year) ** Extracted from National Epidemiological Surveillance of Vaccine-Preventable Diseases (NESVPD) database

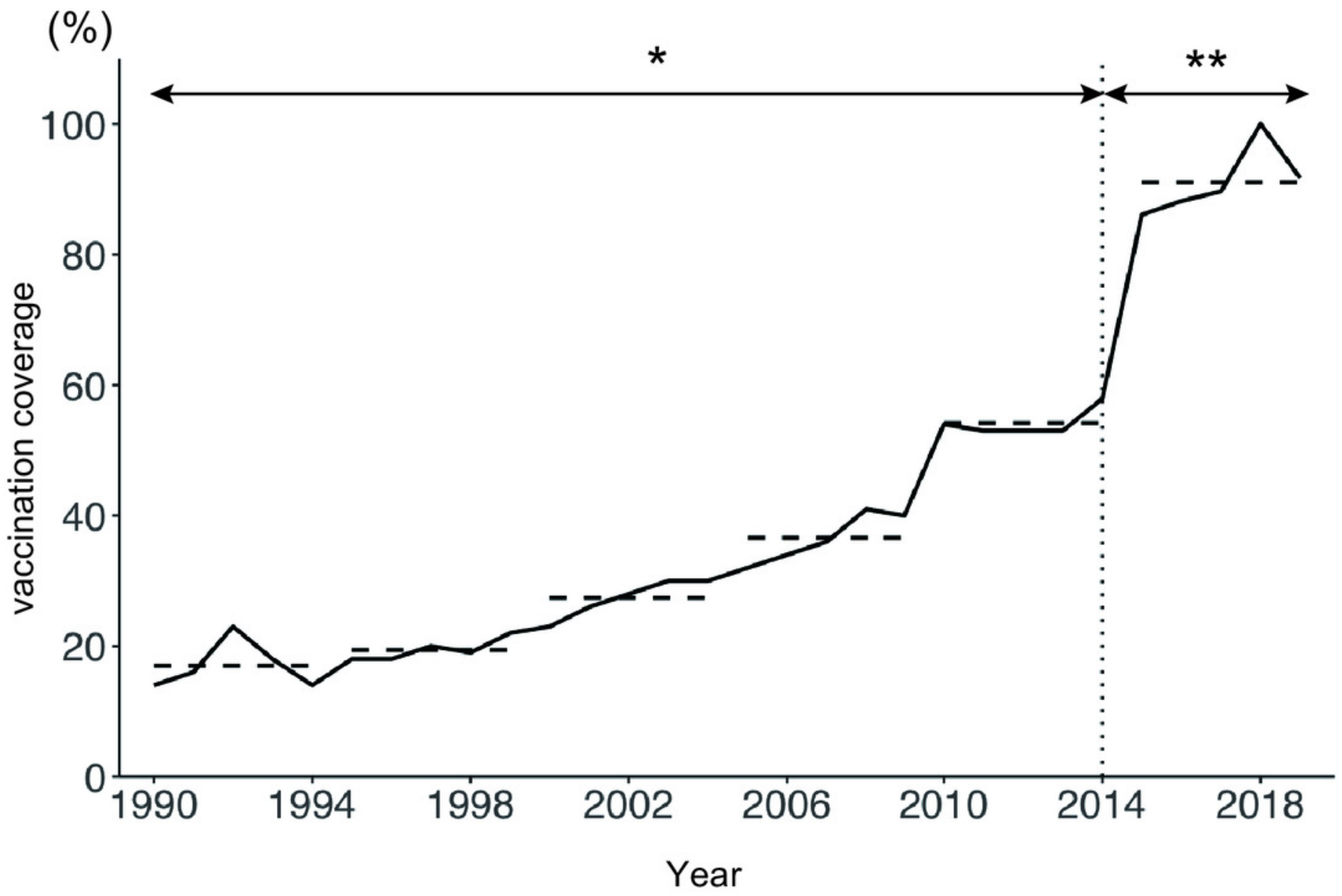


Figure 3

Figure 3: Estimated annual risk of varicella infection among susceptible individuals.

(A) Estimated annual risk of infection stratified by age. (B) Estimated annual risk of infection stratified by year. 

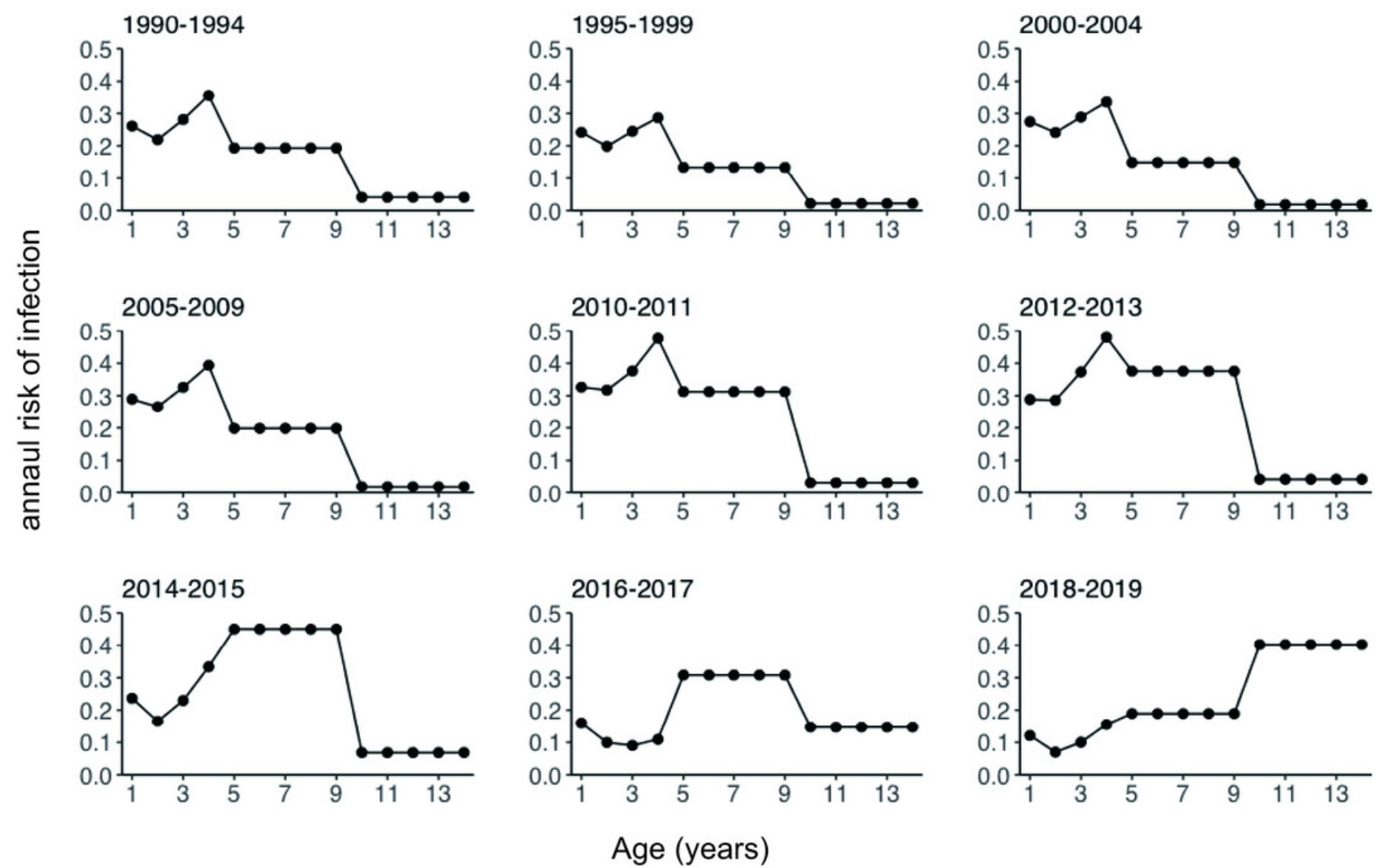
Figure 4

Figure 4: Comparison of observed and estimated numbers of reported varicella cases by age.

Black dots show numbers of reported cases. Blue lines show estimated numbers of reported cases and blue error bars indicate $95 \%$ confidence intervals.
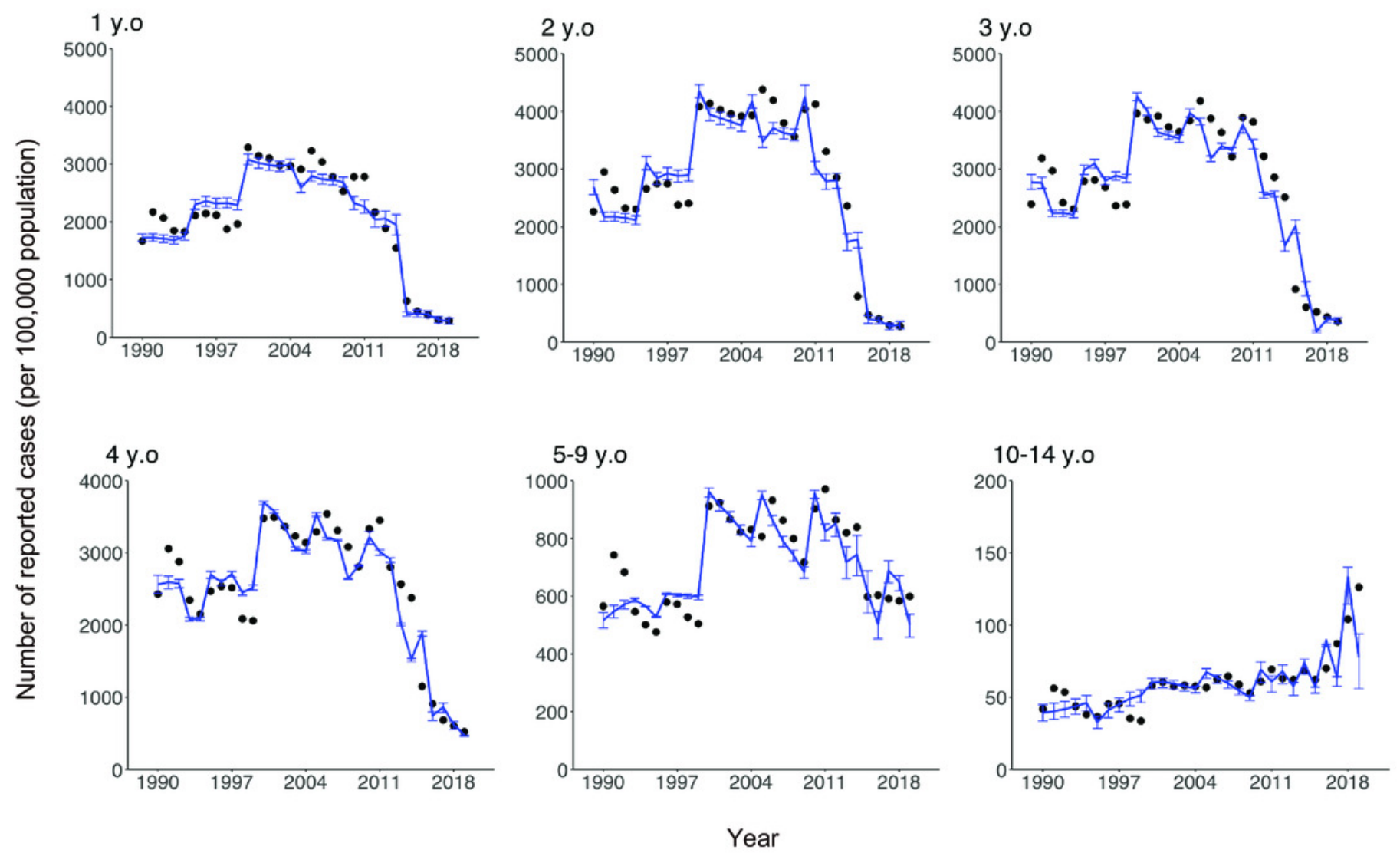
Figure 5

Figure 5: Temporal dynamics of the varicella-susceptible population.

(A) Estimated number of susceptible individuals stratified by birth year. (B) Estimated

proportion of susceptible individuals (estimated number of susceptible individuals/number of newborns in birth cohort) stratified by birth year. (C-E) Estimated number of susceptible individuals by age. Vertical dotted lines indicate the last year for the analyzed datasets (2019).
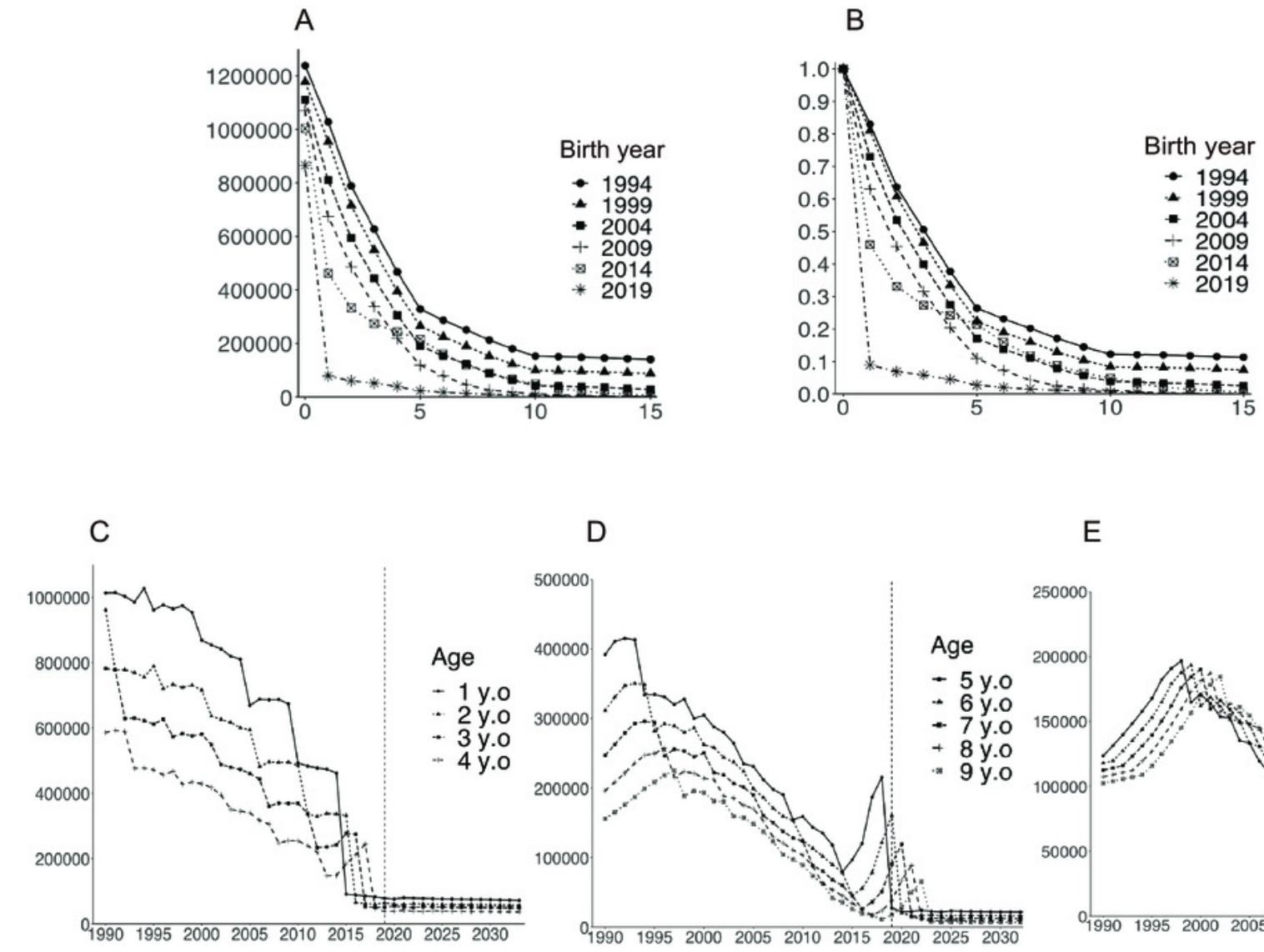

E

0199019952000200520102015202020252030

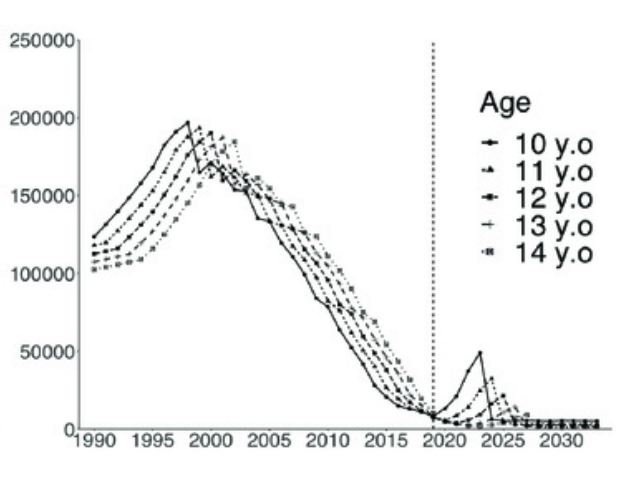

